\title{
The Multiplication Operation of Two Continued Fractions with Positive Non Integer Numerators
}

\author{
${ }^{1}$ Fatima F. Abdullah, ${ }^{2}$ Souad I. Mugassabi \\ ${ }^{1,2}$ University of Benghazi, Benghazi, Libya
}

\begin{abstract}
This paper is a sequel to our previous work in which we found a elementary arithmetic operators of simple continued fractions. We consider the continued fractions (C.F.). We found the multiplication operation of two continued fractions with positive non integer numerators. We apply our finding to many examples of continued fractions.
\end{abstract}

Keywords: Continued fractions. Multiplication operation of continued fractions.

\section{INTRODUCTION}

Since the beginning of the 20th century continued fractions have made their appearances in other field. An example of their versatility: solving Gear-Ratio problem and solving indeterminate equations. Continued fractions have also been utilized within computer algorithms for computing rational approximations to some real number $[1,2,4,5,9]$. Continued fractions have been extensively studied and there is a large body of research related to them $[3,10]$. Our main study is the operations of the continued fractions, previous studies have shown that the operations of the simple continued fractions follow similar patterns to those of continued fractions $[6,7,8]$. Therefore it is important to make this comparison. In this paper, we concentrate on the multiplication operation of two continued fractions with positive non integer numerators. We apply our result form or many examples.

\section{PRELIMINARIES}

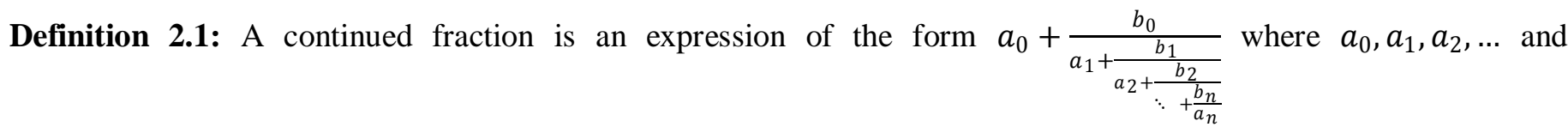
$b_{0}, b_{1}, b_{2}, \ldots$ can be real or complex, and their numbers can be either finite or infinite. In this paper, we consider the continued fractions of the form $a_{0}+\frac{z}{a_{1}+\frac{z}{a_{2}+\frac{z}{\ddots+\frac{z}{a_{n}}}}}$, where $a_{0}$ is an integer, $a_{1}, a_{2}, a_{3}, \ldots, a_{n}$ are positive integer and $z$ is a positive non integer. We denote it by $\left[a_{0} ; a_{1}, a_{2}, \ldots\right]_{z}$.

\section{Example 2.1}
(a) $1+\frac{\frac{5}{2}}{3+\frac{5}{7}}=[1 ; 3,7]_{\frac{5}{2}}$
(b) $-3+\frac{\frac{1}{3}}{1+\frac{1}{2}}=[-3 ; 1,2]_{\frac{1}{3}}$.
(c) $2+\frac{\frac{2}{3}}{1+\frac{\frac{2}{3}}{2+\frac{\frac{2}{3}}{\cdots}}}=[2 ; 1,2, \ldots]_{\frac{2}{3}}$

$(a),(b)$ have a finite number of terms, and $(c)$ has an infinite number of terms.

Theorem 2.1: A number is rational if and only if it can be expressed as a finite C.F. [6]. 
Example 2.2:

(a) $\frac{40}{11}=3+\frac{\frac{3}{2}}{\frac{33}{14}}=3+\frac{\frac{3}{2}}{2+\frac{\frac{3}{2}}{\frac{21}{5}}}=3+\frac{\frac{3}{2}}{2+\frac{\frac{3}{2}}{4+\frac{\frac{3}{2}}{\frac{15}{2}}}}=3+\frac{\frac{3}{2}}{2+\frac{\frac{3}{2}}{4+\frac{\frac{3}{2}}{3+\frac{3}{2}}}}=[3,2,4,7,3]_{3} / 2$.

(b) $\frac{28}{11}=2+\frac{\frac{2}{3}}{\frac{11}{9}}=2+\frac{\frac{2}{3}}{1+\frac{2}{3}}=[2,1,3]_{2} / 3$.

(c) $\frac{3}{7}=0+\frac{\frac{3}{2}}{\frac{7}{2}}=0+\frac{\frac{3}{2}}{3+\frac{3}{3}}=[0,3,3]_{3} / 2$.

(d) $\frac{7}{9}=0+\frac{\frac{7}{9}}{1}=[0 ; 1] 7 / 9$.

Remark 2.1: To expand a negative rational number $-\frac{a}{b}(a, b>0)$ into C.F we take the greatest integer number $\left\lfloor\frac{-a}{b}\right\rfloor$ for the first term of C.F that is, $\left\lfloor\frac{-a}{b}\right\rfloor=a_{o}^{\prime}$, where $-a_{0}^{\prime} \leq-\frac{a}{b}<-a_{1}^{\prime}+1$. We write $-\frac{a}{b}=-a_{0}^{\prime}+\frac{z}{\frac{a^{\prime}}{b^{\prime}}}$ and then we use the same techniques in theorem 2.1 to get the remaining terms for $\frac{a^{\prime}}{b^{\prime \prime}}$ That is, if $\frac{a^{\prime}}{b^{\prime}}=\left[a_{1}^{\prime}, a_{2}^{\prime} \cdots, a_{n}^{\prime}\right]_{Z}$. Then $\frac{-a}{b}=\left[a_{0}^{\prime} ; a_{1}^{\prime}, a_{2}^{\prime} \cdots, a_{n}^{\prime}\right]_{z} .[8]$

Example 2.3: $-\frac{71}{11}=-7+\frac{6}{11}=-7+\frac{\frac{2}{3}}{\frac{11}{9}}=-7+\frac{\frac{2}{3}}{1+\frac{\frac{2}{3}}{3}}=[-7,1,3]_{2 / 3}$.

Lemma 2.1: $\left[c_{0} ; c_{1}, \ldots, c_{j-1}, 0, c_{j+1}, \ldots, c_{n}\right]_{z}=\left[c_{0} c_{1}, \ldots, c_{j-2} c_{j-1}+c_{j+1} c_{j+2} \ldots, c_{n}\right]_{z}$

\section{MULTIPLICATION OPERATION FOR TWO CONTINUED FRACTIONS}

Definition 3.2: Let $\left[a_{0} ; a_{1}, \ldots, a_{m}\right]_{z}$ and $\left[b_{0} ; b_{1}, \ldots, b_{n}\right]_{z}$ be two C.F. $a_{0}, b_{o}$ are non-negative, then we defined their multiplication by:

(1) If $m=n$ then $\left[a_{0} ; a_{1}, \ldots, a_{n}\right]_{z} \times\left[b_{0} ; b_{1}, \ldots, b_{n}\right]_{z}=\left[d_{0} ; d_{1}, \ldots, d_{n}\right]_{z}$

Where

$d_{0}=a_{0} b_{0}$

$d_{1}=\left\lfloor\frac{a_{1} b_{1}}{a_{0} a_{1+} b_{0} b_{1}+z}\right\rfloor$.

$d_{2}=\left\lfloor\frac{z\left(a_{0} b_{2}\left(a_{1} a_{2}+z\right)+a_{2} b_{0}\left(b_{1} b_{2}+z\right)+z a_{2} b_{2}\right)}{\left(a_{1} a_{2}+z\right)\left(b_{1} b_{2}+z\right)-d_{1}\left(a_{o} b_{2}\left(a_{1} a_{2}+z\right)+a_{2} b_{0}\left(b_{1} b_{2}+z\right)+z a_{2} b_{2}\right)}\right\rfloor$. 
$d_{m}=\left\lfloor\frac{z\left(k_{m}\left(a_{1}\right) k_{m}\left(b_{1}\right) k_{m-3}\left(d_{2}\right)-\left(a_{0} k_{m}\left(a_{1}\right) k_{m-1}\left(b_{2}\right)+b_{0} k_{m-1}\left(a_{2}\right) k_{m}\left(b_{1}\right)+z a_{m} b_{m} k_{m-2}\left(a_{2}\right) k_{m-2}\left(b_{2}\right)\right) k_{m-2}\left(d_{1}\right)\right)}{\left(a_{0} k_{m}\left(a_{1}\right) k_{m-1}\left(b_{2}\right)+b_{0} k_{m}\left(b_{1}\right) k_{m-1}\left(a_{2}\right)+z a_{m} b_{m} k_{m-2}\left(a_{2}\right) k_{m-2}\left(b_{2}\right)\right) k_{m-1}\left(d_{1}\right)-k_{m}\left(a_{1}\right) k_{m}\left(b_{1}\right) k_{m-2}\left(d_{2}\right)}\right\rfloor$, if $m$ odd $d_{m}=\left\lfloor\frac{z\left(\left(a_{0} k_{m-1}\left(b_{2}\right) k_{m}\left(a_{1}\right)+b_{0} k_{m-1}\left(a_{2}\right) k_{m}\left(b_{1}\right)+z a_{m} b_{m} k_{m-2}\left(a_{2}\right) k_{m-2}\left(b_{2}\right)\right) k_{m-2}\left(d_{1}\right)-k_{m}\left(a_{1}\right) k_{m}\left(b_{1}\right) k_{m-3}\left(d_{2}\right)\right)}{k_{m}\left(a_{1}\right) k_{m}\left(b_{1}\right) k_{m-2}\left(d_{2}\right)-\left(a_{0} k_{m-1}\left(b_{2}\right) k_{m}\left(a_{1}\right)+b_{0} k_{m-1}\left(a_{2}\right) k_{m}\left(b_{1}\right)+z a_{m} b_{m} k_{m-2}\left(a_{2}\right) k_{m-2}\left(b_{2}\right)\right) k_{m-2}\left(d_{1}\right)}\right\rfloor$, if $m$ even, for $m=2,3, \ldots, n$. The last term $d_{n}$ is to expanded again as a C.F .

(2) If $m \neq n$ then (suppose that $m<n$ ) then

$\left[a_{0} ; a_{1}, \ldots, a_{m}\right]_{z} \times\left[b_{0} ; b_{1}, \ldots, b_{m}, b_{m+1}, \ldots, b_{n}\right]_{z}=\left[d_{0}^{\prime} ; d_{1}^{\prime}, \ldots, d_{m}^{\prime}, d_{m+1}^{\prime}, \ldots, d_{n}^{\prime}\right]_{z} \quad$ (3.2b) where $d_{j}^{\prime}=d_{j}$ for $j=1,2, \ldots, m,\left(d_{j}\right.$ as we did for case $\left.m=n\right)$, while $d_{j}^{\prime}=d_{j, j-m}$

$d_{j, j-m}=\left\lfloor\frac{z\left(k_{m}\left(a_{1}\right) k_{j}\left(b_{1}\right) k_{j-3}\left(d_{2}\right)-\left(a_{0} k_{m}\left(a_{1}\right) k_{j-1}\left(b_{2}\right)+b_{0} k_{j}\left(b_{1}\right) k_{m-1}\left(a_{2}\right)+z k_{m-1}\left(a_{2}\right) k_{j-1}\left(b_{2}\right)\right) k_{j-2}\left(d_{1}\right)\right)}{\left(a_{0} k_{m}\left(a_{1}\right) k_{j-1}\left(b_{2}\right)+b_{0} k_{j}\left(b_{1}\right) k_{m-1}\left(a_{2}\right)+z k_{m-1}\left(a_{2}\right) k_{j-1}\left(b_{2}\right)\right) k_{j-1}\left(d_{1}\right)-k_{m}\left(a_{1}\right) k_{j}\left(b_{1}\right) k_{j-2}\left(d_{2}\right)}\right\rfloor$, if $j$ is odd

$d_{j, j-m}=\left\lfloor\frac{z\left(\left(a_{0} k_{m}\left(a_{1}\right) k_{j-1}\left(b_{2}\right)+b_{0} k_{j}\left(b_{1}\right) k_{m-1}\left(a_{2}\right)+z k_{m-1}\left(a_{2}\right) k_{j-1}\left(b_{2}\right)\right) k_{j-2}\left(d_{1}\right)-k_{m}\left(a_{1}\right) k_{j}\left(b_{1}\right) k_{j-3}\left(d_{2}\right)\right)}{k_{m}\left(a_{1}\right) k_{j}\left(b_{1}\right) k_{j-2}\left(d_{2}\right)-\left(a_{o} k_{m}\left(a_{1}\right) k_{j-1}\left(b_{2}\right)+b_{0} k_{j}\left(b_{1}\right) k_{m-1}\left(a_{2}\right)+z k_{m-1}\left(a_{2}\right) k_{j-1}\left(b_{2}\right)\right) k_{j-1}\left(d_{1}\right)}\right\rfloor, \quad$ if $j \quad$ is $\quad$ even, $\quad$ for $j=m+1, m+2, \ldots, n$. Last term is to be treated as continued fractions as we did before, and not as greatest integer number.

Example 3.1: Find $[3 ; 3]_{3 / 2} \times[3 ; 7]_{3} / 2$

Solution: Let $[3 ; 3]_{3 / 2}=\left[a_{0} ; a_{1}\right]_{z}$ and $[3 ; 7]_{3 / 2}=\left[b_{0} ; b_{1}\right]_{z},(m=n=1)$, from $(3.2 a)$, we have $[3 ; 3]_{3 / 2} \times[3 ; 7]_{3 / 2}=\left[a_{0} ; a_{1}\right]_{z} \times$ $\left[b_{0} ; b_{1}\right]_{z}=\left[d_{o} ; d_{1}\right]_{z}$, where $d_{1}$ is the last term and

$d_{0}=a_{0} b_{0}=3 \cdot 3=9$.

$d_{1}=\frac{a_{1} b_{1}}{a_{0} a_{1}+b_{0} b_{1}+z}=\frac{3 \cdot 7}{3 \cdot 3+3 \cdot 7+\frac{3}{2}}=\frac{2}{3} \quad$ (to be treated as C.F).

$=[0 ; 2,6]_{3} / 2$.

Then $[3 ; 3]_{3 / 2} \times[3 ; 7]_{3 / 2}=[9 ; 0,2,6]_{3} / 2 \quad$ (by lemma 2.1)

$$
=[11 ; 6]_{3} / 2
$$

Example 3.2: Find $[3 ; 3,3]_{3 / 2} \times[6 ; 1,2]_{3 / 2}$.

Solution: Let $[3 ; 3,3]_{3 / 2}=\left[a_{0} ; a_{1}, a_{2}\right]_{3 / 2}$ and $[6 ; 1,2]_{3 / 2}=\left[b_{0} ; b_{1}, b_{2}\right]_{3 / 2}, m=n=2$ from $(3.2 \mathrm{a})$ we have $[3 ; 3,3]_{3 / 2} \times$ $[6 ; 1,2]_{3 / 2}=\left[d_{0} ; d_{1}, d_{2}\right]_{z}$, where $d_{2}$ is the last term and $d_{0}=a_{0} b_{0}=3 \cdot 6=18$. 
$d_{1}=\left\lfloor\frac{a_{1} b_{1}}{a_{0} a_{1}+b_{0} b_{1}+z}\right\rfloor=\left\lfloor\frac{3}{9+6+\frac{3}{2}}\right\rfloor=\left\lfloor\frac{2}{11}\right\rfloor=0$.

$d_{2}=\frac{z\left(a_{0} b_{2}\left(a_{1} a_{2}+z\right)+a_{2} b_{0}\left(b_{1} b_{2}+z\right)+z a_{2} b_{2}\right)}{\left(a_{1} a_{2}+z\right)\left(b_{1} b_{2}+z\right)-d_{1}\left(a_{o} b_{2}\left(a_{1} a_{2}+z\right)+a_{2} b_{0}\left(b_{1} b_{2}+z\right)+z a_{2} b_{2}\right)}$,

$=\frac{\frac{3}{2}\left(3 \cdot 2\left(9+\frac{3}{2}\right)+6 \cdot 3\left(2+\frac{3}{2}\right)+6 \cdot \frac{3}{2}\right)}{\left(9+\frac{3}{2}\right)\left(2+\frac{3}{2}\right)-0\left(3 \cdot 2\left(9+\frac{3}{2}\right)+6 \cdot 3\left(2+\frac{3}{2}\right)+6 \cdot \frac{3}{2}\right)}=\frac{270}{49} \quad$ (to be treated as C.F), we get

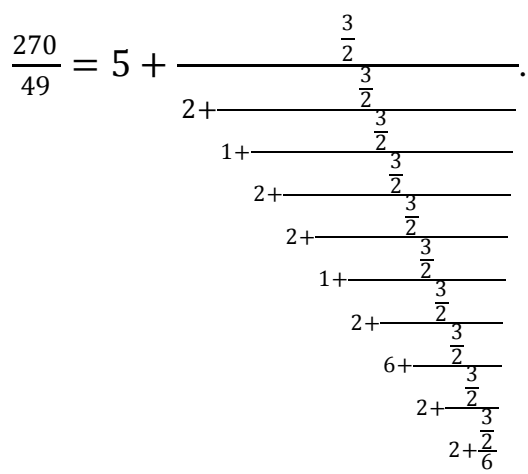

Then $[3 ; 3,3]_{3 / 2} \times[6 ; 1,2]_{3 / 2}=[18 ; 0,5,2,1,2,2,1,2,6,2,2,6]_{3 / 2} \quad$ (by lemma 2.1)

$$
=[23 ; 2,1,2,2,1,2,6,2,2,6]_{3} / 2 .
$$

Example 3.3: Find $[3 ; 3]_{3 / 2} \times[3 ; 3,3]_{3} / 2$

Solution: Let $[3 ; 3]_{3 / 2}=\left[a_{0} ; a_{1}\right]_{z}$ and $[3 ; 3,3]_{3 / 2}=\left[b_{0} ; b_{1}, b_{2}\right]_{z}, m=1, n=2$ from definition (3.2b) we get $[3 ; 3]_{3 / 2} \times$ $[3 ; 3,3]_{3 / 2}=\left[a_{0} ; a_{1}\right]_{z} \times\left[b_{0} ; b_{1}, b_{2}\right]_{z}=\left[d_{0}^{\prime} ; d_{1}^{\prime}, d_{2}^{\prime}\right]_{z}$, where $d_{2}^{\prime}$ is the last term and

$d_{0}^{\prime}=d_{0}=a_{0} b_{0}=3 \cdot 3=9$.

$d_{1}^{\prime}=d_{1}=\left\lfloor\frac{a_{1} b_{1}}{a_{0} a_{1}+b_{0} b_{1}+z}\right\rfloor=\left\lfloor\frac{9}{3 \cdot 3+3 \cdot 3+\frac{3}{2}}\right\rfloor=\left\lfloor\frac{18}{39}\right\rfloor=0$.

$d_{2}^{\prime}=d_{2}=\left\lfloor\frac{z\left(a_{0} a_{1} b_{2}+b_{0}\left(b_{1} b_{2}+z\right)+z b_{2}\right)}{a_{1}\left(b_{1} b_{2}+z\right)-d_{1}\left(a_{0} a_{1} b_{2}+b_{0}\left(b_{1} b_{2}+z\right)+z b_{2}\right)}\right\rfloor=\left\lfloor\frac{\frac{3}{2}\left(27+3\left(9+\frac{3}{2}\right)+\frac{3}{2} \cdot 3\right)}{3\left(9+\frac{3}{2}\right)}\right\rfloor=\left\lfloor\frac{189}{63}\right\rfloor=3$.

Then $[3 ; 3]_{3 / 2} \times[3 ; 3,3]_{3 / 2}=[9 ; 0,3]_{3 / 2} \quad($ by lemma 2.1)

$$
=[12]_{3 / 2}=12 \text {. }
$$

To check, we have $[3 ; 3]_{3 / 2} \times[3 ; 3,3]_{3 / 2}=\frac{7}{2} \cdot \frac{24}{7}=\frac{24}{2}=12$

Example 3.4: Find $[4 ; 2]_{7 / 4} \times[2 ; 9,5,7]_{7 / 4}$. 
Solution: Let $[4 ; 2]_{7 / 4}=\left[a_{0} ; a_{1}\right]_{z},[2 ; 9,5,7]_{7 / 4}=\left[b_{0} ; b_{1}, b_{2}, b_{3}\right]_{z}, m=1, n=3(m<n)$ from definition (3.2b) we have $[4 ; 2]_{7 / 4} \times[2 ; 9,5,7]_{7 / 4}=\left[a_{0} ; a_{1}\right]_{z} \times\left[b_{0} ; b_{1}, b_{2}, b_{3}\right]_{z}=\left[d_{0}^{\prime} ; d_{1}^{\prime}, d_{2}^{\prime}, d_{3}^{\prime}\right]_{z}$, where $d_{3}^{\prime}$ is the last term and

$d_{0}^{\prime}=d_{0}=a_{o} b_{0}=4 \cdot 2=8$

$d_{1}^{\prime}=d_{1}=\left\lfloor\frac{a_{1} b_{1}}{a_{0} a_{1}+b_{0} b_{1}+z}\right\rfloor=\left\lfloor\frac{2 \cdot 9}{4 \cdot 2+2 \cdot 9+\frac{7}{4}}\right\rfloor=\left\lfloor\frac{72}{111}\right\rfloor=0$.

$d_{2}^{\prime}=\left\lfloor\frac{z\left(a_{0} a_{1} b_{2}+b_{0}\left(b_{1} b_{2}+z\right)+z b_{2}\right)}{a_{1}\left(b_{1} b_{2}+z\right)-d_{1}\left(a_{0} a_{1} b_{2}+b_{0}\left(b_{1} b_{2}+z\right) z b_{2}\right)}\right\rfloor=\left\lfloor\frac{\frac{7}{4}\left(4 \cdot 2 \cdot 5+2\left(45+\frac{7}{4}\right)+\left(\frac{7}{4}\right)(5)\right)}{2\left(45+\frac{7}{4}\right)-0\left(4 \cdot 2 \cdot 5+\left(45+\frac{7}{4}\right)+\left(\frac{7}{4}\right)(5)\right)}\right\rfloor=\left\lfloor\frac{3983}{1496}\right\rfloor=2$.

$d_{3}^{\prime}=\frac{z\left(a_{1}\left(b_{1} b_{2} b_{3}+z b_{1}+z b_{3}\right)(1)-\left(a_{0} a_{1}\left(b_{2} b_{3}+z\right)+b_{0}\left(b_{1} b_{2} b_{3}+z b_{1}+z b_{3}\right)(1)+(1)\left(b_{2} b_{3}+z\right) z\right) d_{1}^{\prime}\right)}{\left(a_{0} a_{1}\left(b_{2} b_{3}+z\right)+b_{0}\left(b_{1} b_{2} b_{3}+z b_{1}+z b_{3}\right)(1)+z(1)\left(b_{2} b_{3}+z\right)\right)\left(d_{1}^{\prime} d_{2}^{\prime}+z\right)-a_{1}\left(b_{1} b_{2} b_{3}+z b_{1}+z b_{3}\right)}$

$=\frac{\frac{7}{4}\left(2\left(315+9\left(\frac{7}{4}\right)+(7)\left(\frac{7}{4}\right)\right)\right)}{\left((4)(2)\left(35+\frac{7}{4}\right)+2\left(315+(9)\left(\frac{7}{4}\right)+(7)\left(\frac{7}{4}\right)\right)+\left(35+\frac{7}{4}\right)\left(\frac{7}{4}\right)\right)\left(\frac{7}{4}\right)-(2)\left(315+(9)\left(\frac{7}{4}\right)+(7)\left(\frac{7}{4}\right)\right)(2)}=\frac{224}{85}$.

To be treated as C. F.

$\frac{224}{85}=[2 ; 2,2,5,3,2,1,1,2,22,2,3,1,2,6,4,3,10,5,2,4,5,2,3,8,4,9,5,7] 7 / 4$.

Then $[4 ; 2]_{7 / 4} \times[2 ; 9,5,7]_{7 / 4}$

$=[8 ; 0,2,2,2,2,5,3,2,1,1,2,22,2,3,1,2,6,4,3,10,5,2,4,5,2,3,8,4,9,5,7]_{7} / 4 \quad$ (by lemma 2.1$)$

$=[10 ; 2,2,2,5,3,2,1,1,2,22,2,3,1,2,6,4,3,10,5,2,4,5,2,3,8,4,9,5,7]_{7} / 4$

\section{CONCLUSION}

This paper is the Second part for the operations of the continued fractions with positive non Integer numerators. In the first part, we discovered the definitions of addition and subtractions operations of continued fractions. In this part, we defined the multiplication operation of two continued fractions.

\section{REFERENCES}

[1] Alan Baker (1986). "A concise introduction to theory of numbers". Cambridge University press.

[2] Burton, D. M. (2006). "Elementary number theory". Tata McGraw-Hill Education.

[3] Greene, J., \& Schmieg, J. (2017). "Continued Fractions with Non-Integer Numerators". J. Integer Seq., 20(1), 17-1.

[4] Hardy, G. H., Wright, E. M., Heath-Brown, R., Silverman, J., \& Wiles, A. (2008). "An Introduction to the Theory of Numbers". Oxford University Press.

[5] LeVeque, W. J. (2014). "Fundamentals of Number Theory". Courier Corporation.

[6] Mugassabi S. and Amsheri S. (2020) . "The Multiplication and Division of Simple Continued Fractions". IJISM International Journal of Innovation in Science and Mathematics 8 (3): (2020) 2347-9051.

[7] Mugassabi, Souad I., and A. S. Elmabrok. (2019). "The Power of Simple Continued Fractions." EPHInternational Journal of Applied Science (ISSN: 2208-2182) 1.1: 824-833.

[8] Mugassabi S. and Mistiri F. (2015). "The Elementary Arithmetic Operators of Continued Fractions". Am-Euras. J. Sci.Res., 10 (5): 251 - 263.

[9] C.D. Olds. (1963). "Continued Fractions". 5th Edition, New York: Random House, Inc. 


\section{Citation of this Article:}

Fatima F. Abdullah, Souad I. Mugassabi, "The Multiplication Operation of Two Continued Fractions with Positive Non Integer Numerators" Published in International Research Journal of Innovations in Engineering and Technology - IRJIET, Volume 4, Issue 7, pp 14-19, July 2020. https://doi.org/10.47001/IRJIET/2020.407003 